\title{
gु
}

\section{Formation of Phase Space Holes and Clumps}

\author{
M. K. Lilley \\ Physics Department, Imperial College, London SW7 2AZ, United Kingdom \\ R. M. Nyqvist \\ Department of Earth and Space Sciences, Chalmers University of Technology, 41296 Göteborg, Sweden
} (Received 30 January 2014; published 14 April 2014)

\begin{abstract}
It is shown that the formation of phase space holes and clumps in kinetically driven, dissipative systems is not restricted to the near threshold regime, as previously reported and widely believed. Specifically, we observe hole-clump generation from the edges of an unmodulated phase space plateau, created via excitation, phase mixing and subsequent dissipative decay of a linearly unstable bulk plasma mode in the electrostatic bump-on-tail model. This has now allowed us to elucidate the underlying physics of the holeclump formation process for the first time. Holes and clumps develop from negative energy waves that arise due to the sharp gradients at the interface between the plateau and the nearly unperturbed, ambient distribution and destabilize in the presence of dissipation in the bulk plasma. We confirm this picture by demonstrating that the formation of such nonlinear structures in general does not rely on a "seed" wave, only on the ability of the system to generate a plateau. In addition, we observe repetitive cycles of plateau generation and erosion, the latter due to hole-clump formation and detachment, which appear to be insensitive to initial conditions and can persist for a long time. We present an intuitive discussion of why this continual regeneration occurs.
\end{abstract}

DOI: $10.1103 /$ PhysRevLett.112.155002

PACS numbers: 52.35.-g, 52.25.Dg

Dissipative systems driven far from thermodynamic equilibrium have been of considerable interest over the years, due to their tendency to spontaneously reorganize themselves [1-3]. An important example is a magnetically confined, burning deuterium-tritium plasma, in which fusion born alpha particles maintain a nonthermal distribution that allows them to resonantly destabilize bulk plasma waves that would otherwise be damped by dissipative processes [4]. Such fluctuations are a critical concern since they may subsequently redistribute and potentially eject the fast particles from the plasma prematurely [5].

The nonlinear character of these instabilities plays a large role in determining the fate of the fast particles. They may saturate at low amplitude, in which case the overall effect on the plasma is small, or be bursting in nature with larger amplitude. The latter type is often associated with significant losses of fast particles, cf. [6], and frequently exhibits chirping patterns in the wave frequency away from the normal modes of the bulk plasma. Extensive modeling over the years, in simplified systems [7-10] and in more realistic geometries [11], has revealed that the frequency shifts can be attributed to the formation and subsequent evolution of long-living structures in the fast particle distribution, so called holes (a depletion of particles) and clumps (an excess of particles). The holes and clumps form in the proximity of the wave-particle resonances of a kinetically unstable bulk plasma mode, and once firmly established, they represent nonlinear waves of so-called BGK type [12] whose frequencies are slightly up- and down-shifted with respect to that of the initial instability. Their subsequent convective motion in phase space is synchronized to the change in wave frequency and has previously been successfully described by adiabatic theory [13].

Despite all previous modeling efforts, two fundamental questions have so far remained unanswered, namely, why and how the holes and clumps form. Part of the reason behind this knowledge gap lies in the previously reported result, $[7,14]$, that hole-clump formation only occurs when the background dissipation processes are sufficiently large to compete with the kinetic drive, i.e., when the initial seed wave is only marginally unstable. However, the notion that holes and clumps are near-threshold phenomena has obscured the understanding of the role played by dissipation. In fact, this notion is simply not true. We show in this Letter that any amount of background dissipation results in bursting instabilities, meaning that holes and clumps can be generated far from, as well as close to, the instability threshold. The underlying physics is that holes and clumps develop from negative energy waves, [15], which grow rather than damp as a result of dissipation. Their existence relies on the presence of a nearly unmodulated plateau in the fast particle distribution, whose interface with the surroundings is sharp enough to alter the dielectric response of the fast particles as to support waves near the plateau edge. Such plateau states may arise, e.g., as a result of phase mixing and subsequent dissipative decay of an initially unstable bulk plasma wave, as in experiments. In general, though, an initial seed instability is not a 
requirement, it is only essential that the system has the ability to generate a plateau.

A paradigm for the study of fast particle instabilities, and one which we adopt for our analysis, is the onedimensional, electrostatic bump-on-tail model. Despite the specifics of this idealized model, it captures resonant particle physics in more general, multidimensional systems, [16], such as radial transport of fast particles in tokamaks, [17]. The model considers a purely electrostatic traveling wave, with a single wave number $k$ and spatial period $\lambda=2 \pi / k$, in a uniform plasma of three species. The bulk consists of static background ions and "cold" fluid electrons. The latter respond linearly to the wave field $E$, are subject to a dissipative, collisional, friction force and are characterized by their mass $m_{e}$, equilibrium density $n_{e}$, and perturbed velocity $V$. The third species is a low density population of collisionless fast electrons, whose distribution function $F$ is treated kinetically. The resulting closed system of equations is given by

$$
\begin{gathered}
\frac{\partial F}{\partial t}+v \frac{\partial F}{\partial x}-\frac{|e| E}{m_{e}} \frac{\partial F}{\partial v}=0, \\
\frac{\partial V}{\partial t}=-\frac{|e| E}{m_{e}}-\nu_{c} V, \\
\frac{\partial E}{\partial t}=\frac{|e|}{\epsilon_{0}}\left[n_{e} V+\int\left(F-F_{0}\right) v d v\right],
\end{gathered}
$$

where $\nu_{c}$ is the cold electron collision frequency and $F_{0}(v)$ is the unperturbed distribution of fast electrons. It is assumed that $\nu_{c} \ll \omega_{p}$ and that the perturbed fast particle current is small as compared to that from the background, so that $E=E_{1}(t) \cos \left(k x-\omega_{p} t\right)$ is a good approximation, where $d \ln E_{1} / d t \ll \omega_{p} \equiv \sqrt{n_{e} e^{2} / m_{e} \varepsilon_{0}}$ (see Ref. [10] for details).

The classic instability results from a positive slope, $\partial F_{0} / \partial v>0$, at the resonant velocity $v=\omega_{p} / k$, which causes wave growth at a rate $\gamma_{L}=\left(\omega_{p}^{3} \pi / 2 k^{2} n_{e}\right) \partial F_{0} / \partial v$. Adding weak dissipation, $\gamma_{d} \equiv \nu_{c} / 2<\gamma_{L}$, merely reduces the growth rate to $\gamma_{L}-\gamma_{d}$. After the initial growth, however, the classic and dissipative behaviors diverge. Whereas the classic case ends in wave saturation at $\omega_{B} \equiv \sqrt{e k E_{1} / m_{e}} \approx 3.2 \gamma_{L}$, [18], the dissipative case leads to hole-clump formation and frequency chirping as seen in Fig. 1. Contrary to previous reports, cf. [7], where chirping solutions were found only for $\gamma_{d} / \gamma_{L} \gtrsim 0.4$, Fig. 1 shows frequency chirping for both large and small $\gamma_{d} / \gamma_{L}$, i.e., near and far from the instability threshold. Moreover, we note that holes and clumps are not transient phenomena, as shown by the ongoing activity in both spectrograms. Continuous hole-clump generation was previously observed in near-threshold simulations, [10], and discussed in [19], but its origin was never completely understood. We return to this issue at the end of the present Letter. For now,
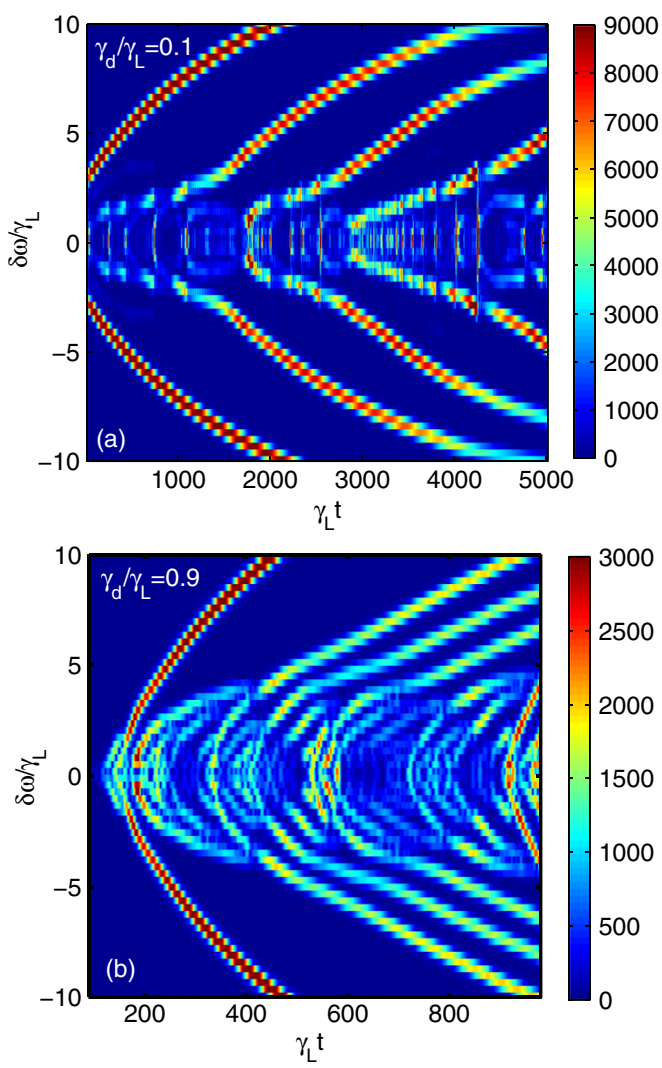

FIG. 1 (color online). Fourier spectrogram of the electric field amplitude $E_{1}$, showing frequency chirping far from (a) and near to (b) the instability threshold. Simulations performed using BOT $[10,20]$.

we focus on the first bursts in Fig. 1 and, in particular, the important observation that in both cases, the chirping initiates with noticeable shifts from $\omega_{p}$, i.e., with nonvanishing $\delta \omega \equiv \omega-\omega_{p}$. This observation is crucial for understanding the hole-clump formation process, as will be described below.

First, consider the limit of small $\gamma_{d} / \gamma_{L}$. Slightly beyond the initial linear growth phase, but prior to chirping, the increasing electric field amplitude $E_{1}$ reaches a maximum where the energy released by phase mixing balances the dissipated power. The associated phase space portrait for the fast particle distribution exhibits an eye-shaped, flattened trapping area [Fig. 2(a)], whose velocity width scales as $\sqrt{E_{1}}$. During the subsequent dissipative decay, trapped particles are released as the separatrix shrinks and begin to stream freely. Since the time scale for the decay is long in the limit of small $\gamma_{d} / \gamma_{L}$, one would expect the final state to be fieldless and exhibit an unmodulated phase space plateau in the fast particle distribution. This is, however, not the case. The amplitude of the bulk mode does decay to zero, but there are sideband oscillations, resulting from incomplete phase mixing, whose amplitudes at first decay but then begin to rise again [Fig. 2(b)] and eventually evolve into chirping modes [Fig. 2(c)]. Although the 

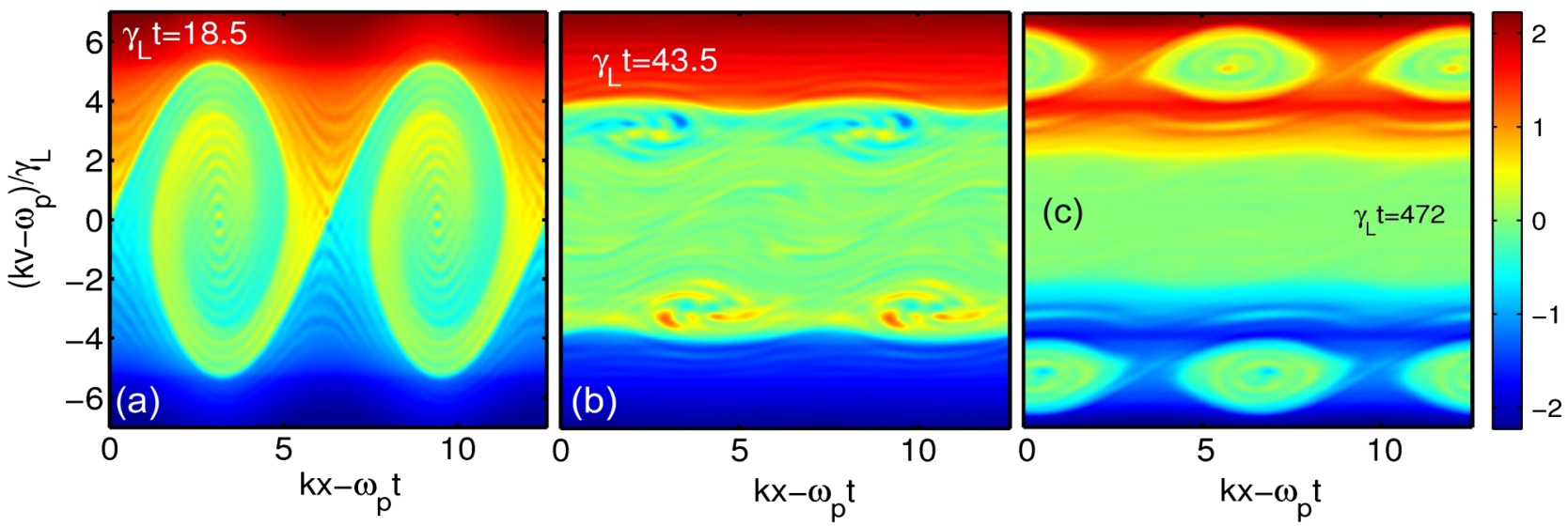

FIG. 2 (color online). Snapshots of the resonant fast particle distribution function for $\gamma_{d} / \gamma_{L}=0.1$ that display (a) the initial phase mixing followed by (b) the almost spatially uniform plateau with sideband trapping regions forming close to the edge, and finally (c) a detaching hole-clump pair. Obtained using BOT [10,20].

distribution tends towards an unmodulated plateau, such a state is actually unstable with respect to modes whose phase velocities are shifted from the initial wave-particle resonance towards the plateau edge, a claim that will be justified shortly. An analogous story can be told for the near threshold case, where dissipation acts overall to produce a similar plateau, albeit in a more sophisticated way. The area of phase space perturbed by the initial plasma wave is small when $\gamma_{d} / \gamma_{L} \lesssim 1$, so the sidebands formed from the incomplete phase mixing interact strongly with the bulk plasma mode. The interaction results in several stages of rise and fall in the wave amplitude, each successively larger, until eventually a plateau establishes from which chirping modes develop [as seen in Fig. 1(b)].

The unstable nature of an unmodulated phase space plateau is demonstrated via linear stability analysis of the shelflike distribution shown in Fig. 3. Following the method of Landau, [21], the time asymptotic behavior of the linearized system is of the form $\exp (-i \omega t)$, where $\omega$ is the solution to

$$
\varepsilon=1-\frac{\omega_{p}^{2}}{\omega\left(\omega+i \nu_{c}\right)}-\frac{|e|^{2}}{m_{e} \varepsilon_{0} k} \int_{-\infty}^{\infty} \frac{\partial F_{0} / \partial v}{k v-\omega} d v=0
$$

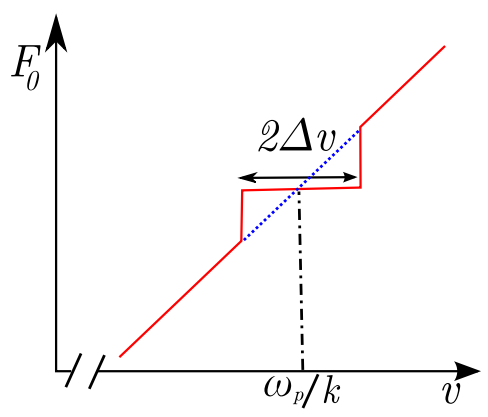

FIG. 3 (color online). Model "shelf" distribution for the fast particles.
The shelf half-width, $\Delta v$, is small compared to $\omega_{p} / k$, meaning $\omega_{R} \approx \omega_{p}$ (where the subscripts $R$ and $I$ denote real and imaginary parts). In this limit, the cold electron contribution to $\varepsilon$ simplifies, and the dispersion relation takes the form

$$
\begin{aligned}
\varepsilon= & \frac{2}{\omega_{p}}\left\{\delta \omega+i \gamma_{d}+\frac{\gamma_{L}}{\pi}[\log [k \Delta v-\delta \omega]-\log [k \Delta v+\delta \omega]\right. \\
& \left.\left.+\frac{2 \delta \omega k \Delta v}{\delta \omega^{2}-(k \Delta v)^{2}}\right]\right\}=0
\end{aligned}
$$

where log denotes the complex logarithm.

A numerical investigation of Eq. (3) reveals three roots with phase velocities within the plateau. The existence of two extra roots beyond that of the classic instability is due, in a beamlike fashion, to the discontinuities in the distribution at the shelf edges. In the absence of dissipation, there is a critical width, given by $k \Delta v=4 \gamma_{L} / \pi$, at which all three roots coalesce at the origin. In fact, the dissipationless case always has a root with $\delta \omega=0$. Below the critical width, it is complemented by a stable-unstable conjugated pair on the imaginary axis (Fig. 4). In this case, instability

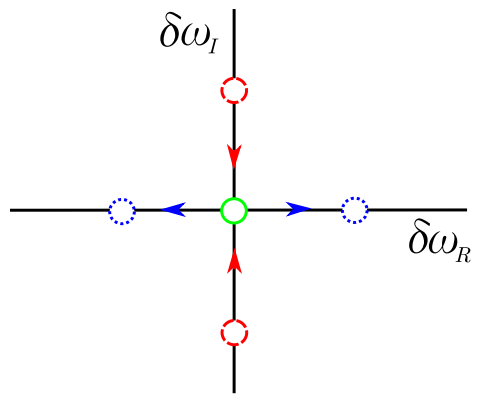

FIG. 4 (color online). Motion of the two nontrivial roots of Eq. (3) for $\gamma_{d}=0$ as the shelf width increases from below (red dashed) to above (blue dotted) the critical value $k \Delta v=4 \gamma_{L} / \pi$. 
reflects the fact that phase mixing from a small perturbation at $\omega=\omega_{p}$ is still a profitable energy release mechanism. Beyond the critical width, phase mixing can no longer support a growing plasma mode, so linear instability is suppressed. More interestingly, the two roots complementary to the plasma mode now diverge along the real axis (Fig. 4). These tendencies can be captured analytically via an expansion around the critical width by setting $k \Delta v=4 \gamma_{L}(1 \pm \delta) / \pi$, with $0<\delta \ll 1$. In addition to the solution $\delta \omega=0$, one then finds two roots that have $\delta \omega_{R}=0, \quad \delta \omega_{I}= \pm i 1.5 \gamma_{L} \sqrt{\delta} \quad$ for the lower and $\delta \omega_{R}= \pm 1.5 \gamma_{L} \sqrt{\delta}, \delta \omega_{I}=0$ for the upper sign. The diverging roots behave most interestingly when dissipation is included. Whereas the plasma mode with $\delta \omega_{R}=0$ damps with $\delta \omega_{I} \sim-\gamma_{d}$, these modes actually destabilize weakly, with $0<\delta \omega_{I} \ll \delta \omega_{R}$. In the large plateau limit, the roots approach the discontinuities in the distribution function, $|\delta \omega| / k \Delta v \rightarrow 1$, so this feature can be explicitly seen by expanding Eq. (3) to lowest order in $k \Delta v-\delta \omega$, giving

$$
\begin{gathered}
\delta \omega_{R} \approx \pm k \Delta v\left[1-\frac{\gamma_{L}}{\pi} \frac{k \Delta v}{(k \Delta v)^{2}+\gamma_{d}^{2}}\right], \\
\delta \omega_{I} \approx \gamma_{d} \frac{\gamma_{L}}{\pi} \frac{k \Delta v}{(k \Delta v)^{2}+\gamma_{d}^{2}} .
\end{gathered}
$$

Further to the linear stability analysis, Fig. 5 displays snapshots from a nonlinear simulation, initialized with a large, shelflike plateau, where velocity modulations just inside the plateau edges [at shifts predicted by Eq. (4a) to within $3 \%$ ] indeed evolve into chirping holes and clumps on a time scale consistent with Eq. (4b). In this simulation, the dissipation is large enough to completely suppress the

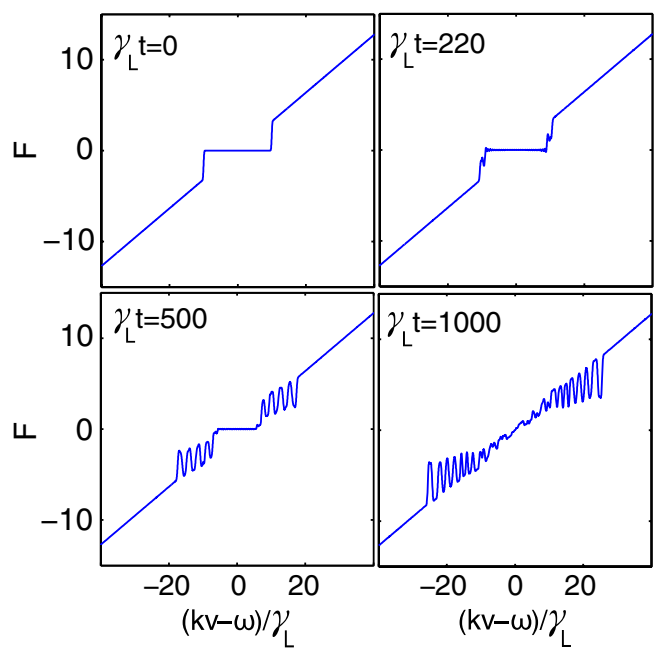

FIG. 5 (color online). Spatially averaged distribution function evolved using the BOT code [10,20] for $\gamma_{d} / \gamma_{L}=2, k \Delta v / \gamma_{L}=$ 10 and initial normalized amplitude $\omega_{B}^{2} / \gamma_{L}^{2}=10^{-6}$. The unstable plateau generates holes and clumps that eventually completely erode the plateau state. bulk plasma mode, $\gamma_{d} / \gamma_{L}=2$, emphasizing its lack of involvement in the hole-clump formation process.

The physical reason for the instability of the edge modes is that they are negative energy waves that grow in the presence of dissipation rather than being abated. This can be seen by evaluating the wave energy, which in the electrostatic 1D case is [22],

$$
W=\frac{\varepsilon_{0}}{4}|E|^{2} \frac{\partial}{\partial \omega}\left(\omega \epsilon_{h}\right)
$$

where $\varepsilon_{h}$ is the Hermitian part of the dielectric constant evaluated at real frequency $\omega$ [i.e., $\varepsilon$ from Eq. (3), without $\left.i \gamma_{d}\right]$. Then the condition for negative wave energy becomes $\partial \varepsilon_{h} / \partial \omega<0$, or

$$
\frac{k \Delta v}{\gamma_{L}}\left[1-\frac{\delta \omega^{2}}{k^{2} \Delta v^{2}}\right]^{2}<\frac{4}{\pi}
$$

It can be verified that the shifted roots of the dispersion relation [Eq. (3)] all satisfy Eq. (6). In particular, in the large shelf limit, for which $|\delta \omega| / k \Delta v \rightarrow 1$, Eq. (6) is clearly satisfied. While one might naively expect the opposite for a near-critical width, the scaling $\delta \omega \sim \sqrt{\delta}$ in fact ensures that the roots shift fast enough to maintain negative energy.

We are now in a position to address the nontransient nature of the bursts in Fig. 1. In general, the first holeclump pair leaves behind a somewhat smaller plateau, which is nevertheless still unstable. If it is large enough, a secondary pair will develop and detach, much like in Fig. 5. This process continues until nonlinear mode interaction within the plateau can no longer be neglected, at which point the system enters a phase during which a large plateau is replenished. The rebuild phase is, however, sophisticated and includes several stages that are outside the validity range of the shelflike plateau model. Nevertheless, some robust features can be extracted from the simulations and interpreted by means of the model, and they depend intrinsically on the value of $\gamma_{d}$. For $\gamma_{d} / \gamma_{L} \ll 1$, the mode interaction is sufficiently strong to destabilize the central plasma mode. The ensuing phase mixing and dissipative damping creates a slightly larger plateau, much as in Fig. 2. This course of events then repeats, thereby gradually enlarging the plateau until it is wide enough that the erosion sequence can begin again [cf. Fig. 1(a), $\gamma_{L} t<1500$ ]. Close to the threshold, on the other hand, the linear shelf model implies that the central mode is heavily damped and, accordingly, seldom destabilizes. Rather, the reconstitution process consists of several false starts where holes and clumps attempt to peel off the small plateau but quickly decay, thereby leaving behind a larger flattened area [cf. Fig. 1(b), $220<\gamma_{L} t<400$ ]. We speculate that the holes and clumps are unable to fully develop because of the strong interaction with the heavily damped central mode, a feature that was also seen to be prominent during the 
formation of the initial plateau. More work is however required in order to assess whether it is possible to construct a simple description of this nonlinear process.

In summary, we have explained the origin and continual production of holes and clumps in terms of the sequential formation and instability of phase space plateaus, arising as a result of phase mixing and dissipative damping of an unstable kinetic resonance. Both near and far from the instability threshold, the holes and clumps develop from negative energy modes that resonate with particles close to the plateau edge, giving credence to the interpretation that the considered mechanism is universal.

The authors wish to thank H. L. Berk for stimulating discussions that helped inspire this Letter.

*m.lilley05@imperial.ac.uk

[1] A. Turing, Phil. Trans. R. Soc. B 237, 37 (1952).

[2] H. Bénard, Ph.D. dissertation, University of Paris, 1900.

[3] I. Prigogine, Introduction to Thermodynamics of Irreversible Processes, (John Wiley, New York, 1967).

[4] W. W. Heidbrink, Phys. Plasmas 15, 055501 (2008).

[5] A. Fasoli et al., Nucl. Fusion 47, S264 (2007).

[6] K. L. Wong et al., Phys. Rev. Lett. 66, 1874 (1991).

[7] H. L. Berk, B. N. Breizman, and N. V. Petviashvili, Phys. Lett. A 234, 213 (1997).

[8] R. G. L. Vann, H. L. Berk, and A. R. Soto-Chavez, Phys. Rev. Lett. 99, 025003 (2007).
[9] M. Lesur, Y. Idomura, and X. Garbet, Phys. Plasmas 16, 092305 (2009).

[10] M. K. Lilley, B. N. Breizman, and S. E. Sharapov, Phys. Plasmas 17, 092305 (2010).

[11] S. D. Pinches et al., Plasma Phys. Controlled Fusion 46, S47 (2004).

[12] I. B. Bernstein, J. Greene, and M. Kruskal, Phys. Rev. 108, 546 (1957).

[13] R. M. Nyqvist, and B. N. Breizman, Phys. Plasmas 20, 042106 (2013).

[14] H. L. Berk, B. N. Breizman, J. Candy, M. Pekker, and N. V. Petviashvili, Phys. Plasmas 6, 3102 (1999).

[15] B. B. Kadomtsev et al., Sov. Phys. JETP 20, 1517 (1965).

[16] B. V. Chirikov, Phys. Rep. 52, 263 (1979).

[17] B. N. Breizman, H. L. Berk, M. S. Pekker, F. Porcelli, G. V. Stupakov, and K. L. Wong, Phys. Plasmas 4, 1559 (1997).

[18] See National Technical Information Service Document No. AD730123 PGG-93. B. Fried, C. Lui, W. Means, and R. Sagdeev, University of California Report No. AD730123 PGG-93, 1971. Copies may be ordered from the National Technical Information Service, Springfield, Virginia 22161.

[19] M. K. Lilley, and B. N. Breizman, Nucl. Fusion 52, 094002 (2012).

[20] M. K. Lilley (2010), source code available from code.google .com/p/bump-on-tail.

[21] L. D. Landau, J. Phys. 10, 25 (1946).

[22] L. D. Landau and E. M. Lifshitz, Electrodynamics of Continuous Media (Pergamon Press, Oxford, 1984), second edition, p. 275. 